\title{
On Computing the Discriminant of an Algebraic Number Field
}

\author{
By Theresa P. Vaughan
}

\begin{abstract}
Let $f(x)$ be a monic irreducible polynomial in $\mathbf{Z}[x]$, and $\mathbf{r}$ a root of $f(x)$ in $\mathbf{C}$. Let $K$ be the field $\mathbf{Q}(\mathbf{r})$ and $\mathscr{R}$ the ring of integers in $K$. Then for some $k \in \mathbf{Z}$, disc $\mathbf{r}=k^{2} \operatorname{disc} \mathscr{R}$. In this paper we give constructive methods for (a) deciding if a prime $p$ divides $k$, and (b) if $p \mid k$, finding a polynomial $g(x) \in \mathbf{Z}[x]$ so that $g(x) \not \equiv 0(\bmod p)$ but $g(\mathbf{r}) / p \in \mathscr{R}$.
\end{abstract}

1. Introduction. Let $f(x)$ be a monic irreducible polynomial with integral coefficients, and $\mathbf{r}$ a root of $f(x)$ in $\mathbf{C}$. Let $K$ be the field $\mathbf{Q}(\mathbf{r})$ and $\mathscr{R}$ the ring of algebraic integers in $K$. It is well-known that

$$
N\left(f^{\prime}(\mathbf{r})\right)=\operatorname{disc} \mathbf{r}=k^{2} \operatorname{disc} \mathscr{R}
$$

for some integer $k$. While $N\left(f^{\prime}(\mathbf{r})\right)$ can be found by straightforward (if tedious) computation, the value of $k$ is quite another story. According to [2, p. 77] for example, to determine $k$, one would have to test a finite number (which may be very large) of elements of $K$ to see if they are integral.

In this paper, we reduce some of the difficulties involved in finding $k$ to more manageable size; our methods do not require a search process. Consider the following problems:

(I) Given a prime $p$ with $p^{2} \mid$ disc $\mathbf{r}$, how can one tell whether or not $p \mid k$ ?

(II) Suppose $p \mid k$. Then, it is known there exists an element $\beta \in K, \beta=g(\mathbf{r})$ for $g(x) \in \mathbf{Z}[x]$ and $g(x) \not \equiv 0(\bmod p)$ such that $\beta / p \in \mathscr{R}$. Construct such an element $\beta$.

A reasonable solution to these problems is furnished by Theorems 5.4, 5.7 and 5.9; it may be summarized as follows:

Suppose that $f(x)$ has degree $n$, and that $p^{2} \mid \operatorname{disc} \mathbf{r}$. Factor $f(x) \bmod p$ :

$$
f(x)=\prod_{i=1}^{r} f_{i}(x)^{e_{i}} \quad\left(f_{i}(x) \text { irreducible }\right) .
$$

If all the $e_{i}=1$, then $p+k$. If any $e_{i}>1$, then let $C$ be the companion matrix of $f(x)$ and compute $f_{i}(C)\left(\bmod p^{2}\right)$. This matrix represents a homogeneous system of linear equations $\bmod p^{2}$; if this system has a nontrivial solution $\bmod p^{2}$, then $p \mid k$ and Theorem 5.9 enables the construction of a $\beta$ as in (II) above. If for each $e_{i}>1$, the system of equations has no nontrivial solution, then $p+k$. The actual labor involved, amounts to the computation and row-reduction $\left(\bmod p^{2}\right)$ of no more than $r n \times n$ matrices.

Received July 20, 1984.

1980 Mathematics Subject Classification. Primary 12A50. 
In Section 2, we give the notation we need from number theory. In Section 3, we give our matrix-theoretic notation, and define one of our basic tools: an Abelian group, associated with an $n \times n$ integral matrix $A$ :

$$
R(A)=\left\{\bar{v} \in \mathbf{Q}^{n}: 0 \leqslant v_{i}<1, A \bar{v} \in \mathbf{Z}^{n}\right\}
$$

whose operation is addition modulo 1 on the coordinates of vectors.

In Section 4, we consider the companion matrix $C$ of $f(x)$ as a linear transformation of $\mathbf{Z}_{p}^{n}$ ( $p$ prime); we then find that the $p$-component of a group $R(g(C))$ for $g(x) \in \mathbf{Z}[x]$, has a particularly nice sort of basis, which then determines (among other things) the arrangement of powers of $p$ on the diagonal of the Smith form for $g(C)$.

In Section 5, we use the machinery developed in Section 4 to answer (I) and (II) above; in Section 6 we give some results which are helpful in computations, and two examples.

Finally, in Section 7, we list some unanswered questions and conjectures.

2. Notation (number-theoretic). Let $f(x) \in \mathbf{Z}[x]$ be monic and irreducible of degree $n$ :

$$
f(x)=x^{n}-a_{n-1} x^{n-1}-a_{n-2} x^{n-2}-\cdots-a_{1} x-a_{0} \quad\left(a_{i} \in \mathbf{Z}\right)
$$

and let $\mathbf{r}$ be a root of $f(x)$ in $\mathbf{C}$. Let $K$ be the field $\mathbf{Q}(\mathbf{r})$ and $\mathscr{R}$ the ring of algebraic integers in $K$. Let $\sigma_{1}, \sigma_{2}, \ldots, \sigma_{n}$ be the embeddings of $K$ in C. If $\alpha \in K$, then the norm and trace of $\alpha$ are defined by $\mathscr{N}(\alpha)=\prod_{i=1}^{n} \sigma_{i}(\alpha) ; \operatorname{Tr}(\alpha)=\sum_{i=1}^{n} \sigma_{i}(\alpha)$.

If $\left\{\alpha_{1}, \alpha_{2}, \ldots, \alpha_{n}\right\} \subset \mathscr{R}$ and if $\mathscr{R}=\left\{\sum_{i=1}^{n} n_{i} \alpha_{i} \mid n_{i} \in \mathbf{Z}\right\}$ then $\left\{\alpha_{1}, \alpha_{2}, \ldots, \alpha_{n}\right\}$ is said to be an integral basis for $\mathscr{R}$. Then the discriminant of $\mathscr{R}$ is given by the square of the determinant of $\left(\sigma_{i}\left(\alpha_{j}\right)\right)$;

$$
\operatorname{disc} \mathscr{R}=\left|\sigma_{i}\left(\alpha_{j}\right)\right|^{2} \in \mathbf{Z} .
$$

Since $\mathbf{r}$ is a root of $f(x)$, the set $\left\{1, \mathbf{r}, \mathbf{r}^{2}, \ldots, \mathbf{r}^{n-1}\right\}$ is a basis for $K$ over $\mathbf{Q}$, but not necessarily an integral basis for $\mathscr{R}$, that is, it is possible that $\mathbf{Z}[\mathbf{r}] \neq \mathscr{R}$ (of course $\mathbf{Z}[\mathbf{r}] \subseteq \mathscr{R}$ always).

Define the discriminant of $\mathbf{r}$ as

$$
\operatorname{disc} \mathbf{r}=\left|\boldsymbol{\sigma}_{i}\left(\mathbf{r}^{j-1}\right)\right|^{2}
$$

one has $\operatorname{disc} \mathbf{r}=k^{2} \operatorname{disc} \mathscr{R}$ for some $k \in \mathbf{Z}$; also $\operatorname{disc} \mathbf{r}=\mathscr{N}\left(f^{\prime}(\mathbf{r})\right)$.

It is well-known that if $p+k$, then the factorization of the ideal $(p)$ in $\mathscr{R}$, into prime ideals, may be determined as follows: Write

$$
f(x) \equiv \prod_{i=1}^{r} f_{i}(x)^{e_{i}}(\bmod p)
$$

and let $P_{i}$ be the ideal $\left(f_{i}(\mathbf{r}), p\right)$. Then $(p)=\prod_{i=1}^{r} P_{i}^{e_{i}}$ is the prime factorization of $(p)$.

On the other hand, if $p \mid k$, then there exists $\beta \in \mathscr{R}$ of the form

$$
\beta=\sum_{i=0}^{n-1} n_{i} \mathbf{r}^{i} \quad\left(n_{i} \in \mathbf{Z}\right),
$$


where not all the $n_{i} \equiv 0(\bmod p)$ and $(1 / p) \beta \in \mathscr{R}$; the factorization $(1)$ does not yield the prime factorization of $(p)$.

As we shall see, however, the factorization (1) may still yield partial information. Our methods rely heavily on matrix representations, described in the next section.

3. Notation (matrix-theoretic). Let $C$ be the companion matrix for $f(x)$ :

$$
C=\left[\begin{array}{ccccc}
0 & 0 & \ldots & 0 & a_{0} \\
1 & 0 & \ldots & 0 & a_{1} \\
0 & 1 & \ldots & 0 & a_{2} \\
0 & . & \ldots & 1 & a_{n-1}
\end{array}\right] .
$$

The minimum and characteristic polynomials of $C$ are both equal to $f(x)$, and $C$ represents the $\mathbf{Q}$-linear map on $K$ "multiplication by $\mathbf{r}$ " in the basis $\left\{1, \mathbf{r}, \mathbf{r}^{2}, \ldots, \mathbf{r}^{n-1}\right\}$ for $K$ over $\mathbf{Q}$. That is, if $\alpha, \beta \in K$ and

$$
\alpha=\sum_{i=0}^{n-1} c_{i} \mathbf{r}^{i} ; \quad \beta=\mathbf{r} \alpha=\sum_{i=0}^{n-1} b_{i} \mathbf{r}^{i} \quad\left(c_{i}, b_{i} \in \mathbf{Q}\right),
$$

then $C \cdot \operatorname{col}\left(c_{0}, \ldots, c_{n-1}\right)=\operatorname{col}\left(b_{0}, \ldots, b_{n-1}\right)$.

One has $\mathbf{Q}(\mathbf{r}) \cong \mathbf{Q}[C]$ and $\mathbf{Z}[\mathbf{r}] \cong \mathbf{Z}[C]$ via the usual correspondence $g(\mathbf{r}) \approx g(C)$. The eigenvalues of $g(C)$ are $\left\{\sigma_{i}(g(\mathbf{r}))\right\},|g(C)|=\mathscr{N}(g(\mathbf{r}))$ and $\operatorname{Tr}(g(C))=\operatorname{Tr}(g(\mathbf{r}))$. Since $f(x)$ is irreducible over $\mathbf{Q}$, any matrix $X=g(C)(g(x) \in \mathbf{Q}[x])$ is singular if and only if it is 0 ; also if $X C=C X$ then $X=g(C)$ and conversely.

We shall need the Smith form $S(X)$ of an integral matrix $X \in \mathbf{Z}_{n \times n}$ with $|X| \neq 0$. For each such $X$, there exist matrices $P, Q$ in $\mathbf{Z}_{n \times n}$ with $|P|= \pm 1$ and $|Q|= \pm 1$ and positive integers $d_{i}$ with $d_{i} \mid d_{i+1}$ for $i=1,2, \ldots, n-1$ such that

$$
P X Q=\left[\begin{array}{llll}
d_{1} & & & 0 \\
& d_{2} & & \\
& & \ddots & \\
0 & & & d_{n}
\end{array}\right]=S(X) .
$$

The $d_{i}$ are called the invariant factors of $X$. If $p^{r_{i}} \| d_{i}$ (that is, $p^{r_{i}} \mid d_{i}$ and $p^{r_{i}+1}+d_{i}$ ) for a prime $p$, then we put

$$
S_{p}(X)=\operatorname{diag}\left(p^{r_{1}}, p^{r_{2}}, \ldots, p^{r_{n}}\right),
$$

and call this the $p$-Smith form of $X$.

Finally, we shall require the following group associated with $X \in \mathbf{Z}_{n \times n},|X| \neq 0$ (see [1] for a detailed discussion).

$$
R(X)=\left\{\bar{v}=\operatorname{col}\left(v_{1}, v_{2}, \ldots, v_{n}\right) \in \mathbf{Q}^{n}: 0 \leqslant v_{i}<1 \text { and } X \bar{v} \in \mathbf{Z}^{n}\right\} .
$$

The operation is addition modulo 1 on the coordinates of vectors. It is proved in [1] that $R(X)$ is an Abelian group with invariant factors $d_{1}, \ldots, d_{n}$; that is, $R(X) \cong$ $C\left(d_{1}\right) \oplus \cdots \oplus C\left(d_{n}\right)$. The order of $R(X)$ is $|X|$. The $p$-component of $R(X)$ (elements whose order is a power of $p$ ) for a prime $p$, is the set of all $\bar{v} \in R(X)$ such that

$$
\bar{v}=\frac{1}{p^{t}} \bar{a} \quad \text { where } t>0 \text { and } \bar{a} \in \mathbf{Z}^{n} .
$$

If $\bar{a} \not \equiv 0(\bmod p)$, then $p^{t}$ is the order of $\bar{v}$ in $R(X)$, and we say that $\bar{v}$ is a $p^{t}$-point for $X$. 
4. Preliminary Results. This section is mostly concerned with the properties of the $p$-component $R_{p}(X)$ of the group $R(X)$, for $X=g(C) \in \mathbf{Z}[C]$ (where $p$ is a prime dividing $|X|)$. From now on, unless otherwise indicated, all polynomials are in $\mathbf{Z}[x]$, all matrices are in $\mathbf{Z}[C], p$ is a prime, and we shall freely employ the following abuse of notation: If $\bar{a} \in \mathbf{Z}^{n}$, we say also $\bar{a} \in \mathbf{Z}_{p}^{n}$, meaning the reduced form of $\bar{a}$ modulo $p$; if $\bar{a} \in \mathbf{Q}^{n}$ we say $\bar{a} \in R(X)$, meaning the reduced form of $\bar{a}$ modulo 1 .

Any integral matrix $X \in \mathbf{Z}_{n \times n}$ may be regarded as a linear transformation of the vector space $\mathbf{Z}_{p}^{n}$, and we use the notation $\operatorname{ker}_{p} X, \operatorname{Im}_{p} X, p$-rank, $p$-nullity, etc., in this setting.

Let $p$ be a fixed prime, and $g(x) \in \mathbf{Z}[x], X=g(C)$. For convenience, we state the following well-known facts and observations as a theorem. (For details see, e.g., [3].)

4.1. THEOREM. (a) $|X| \equiv 0(\bmod p)$ if and only if $X$ is singular on $\mathbf{Z}_{p}^{n}$; (b) $|X| \equiv 0$ $(\bmod p)$ if and only if $\operatorname{gcd}(f(x), g(x)) \neq 1(\bmod p) ;(c) \operatorname{dim}\left(\operatorname{ker}_{p} X\right)=p$-nullity of $X=$ number of invariant factors of $X$ divisible by $p ;(\mathrm{d}) \operatorname{dim}\left(\operatorname{Im}_{p} X\right)=p$-rank $X=n-\left(p\right.$-nullity); (e) Every $C$-invariant subspace $W$ of $\mathbf{Z}_{p}^{n}$ has a cyclic vector $\bar{v}$ for $C$, that is, $W$ has a basis of the form $\left\{\bar{v}, C \bar{v}, C^{2} \bar{v}, \ldots, C^{k-1} \bar{v}\right\}$ where $k=\operatorname{dim} W$; (f) If $W$ is a C-invariant subspace of $\mathbf{Z}_{p}^{n}$, then $W=\operatorname{ker}_{p} k(C)$, where $k(x) \in \mathbf{Z}[x]$ and $k(x) h(x)=f(x)(\bmod p)$ for some $h(x) ; \operatorname{dim} W=$ degree $k(x) ;$ the minimum polynomial for the restriction $C \mid W$ is $k(x)$; and $W=\operatorname{Im}_{p} h(C)$; (g) Let $f(x)=$ $\prod_{i=1}^{r} f_{i}(x)^{e_{i}}(\bmod p)$, where $f_{i}(x)$ is irreducible over $\mathbf{Z}_{p}$ of degree $k_{i}$; and put $W_{i}^{t}=\operatorname{ker}_{p} f_{i}(C)^{t}$. If $e_{i}>1$, then

$$
W_{i}^{1} \subsetneq W_{i}^{2} \subsetneq \cdots \subsetneq W_{i}^{e}=W_{i}^{e_{i}}=\cdots \text {. }
$$

If $X=g(C)$, where

$$
\operatorname{gcd}(f(x), g(x))=\prod_{i=1}^{r} f_{i}(x)^{t_{i}},
$$

then $\operatorname{ker}_{p} X$ is the direct sum of subspaces:

$$
\operatorname{ker}_{p} X=W_{1}^{t_{1}} \oplus W_{2}^{t_{2}} \oplus \cdots \oplus W_{r}^{t_{r}}
$$

Proof. Parts (a)-(d) are obvious; parts (e)-(g) follow from the fact that the minimum and characteristic polynomials of $C$ are equal.

4.2. Definition. Let $X=g(C)$ and let $V \neq\{0\}$ be a subset of $\operatorname{ker}_{p} X$. The $V$-component of $R_{p}(X)$ is the set

$$
R_{V}(X)=\left\{\bar{v} \in R_{p}(X): \bar{v}=\left(1 / p^{t}\right) \bar{a}, \bar{a} \in \mathbf{Z}^{n}, \bar{a} \not \equiv 0(\bmod p) \text { and } \bar{a} \in V\right\} .
$$

Before the main theorem, we need a few lemmas.

4.3. Lemma. Let $X=g(C), g(x) \in \mathbf{Z}[x]$. If there is a vector $\bar{a} \not \equiv 0, \bar{a} \in \operatorname{ker}_{p} X$, then for some $t>0,\left(1 / p^{t}\right) \bar{a} \in R_{p}(X)$. Conversely, if $\left(1 / p^{t}\right) \bar{a} \in R_{p}(X)$, where $\bar{a} \not \equiv$ and $t>0$, then $\bar{a} \in \operatorname{ker}_{p} X$.

Proof. $X \bar{a} \equiv 0(\bmod p)$ if and only if $X \bar{a}=p^{t} \bar{b}$ for some $t>0, \bar{b} \in \mathbf{Z}^{n}$; hence if and only if $X\left(1 / p^{t}\right) \bar{a}=\bar{b} \in \mathbf{Z}^{n}$, that is, $\left(1 / p^{t}\right) \bar{a} \in R_{p}(X)$.

4.4. LEMMA. The following are equivalent:
(a) $\operatorname{ker}_{p} X \cap W_{i} \neq\{0\}$;
(b) $W_{i} \subseteq \operatorname{ker}_{p} X$;
(c) $(1 / p) W_{i} \subseteq R_{p}(X)$;
(d) $f_{i}(x) \mid g(x)(\bmod p)$. 
Proof. Since $f_{i}(x)$ is irreducible $\bmod p, W_{i}=\operatorname{ker}_{p} f_{i}(C)$ has no proper nontrivial $C$-invariant subspaces by Theorem 4.1(f); hence (a) $\leftrightarrow$ (b). The implications (b) $\leftrightarrow$ (d) also follow from Theorem 4.1(f), and (b) $\leftrightarrow$ (c) from Lemma 4.3.

4.5. Lemma. Let $A, B, X, Y, Z \in \mathbf{Z}[C]$ and suppose that $V$ is a subset of $\operatorname{ker}_{p} Z$. If $R_{V}(Z) \subseteq R_{p}(X)$ and $R_{V}(Z) \subseteq R_{p}(Y)$, then $R_{V}(Z) \subseteq R_{p}(A X+B Y)$.

Proof. Let $\bar{v} \in R_{V}(Z)$. By assumption, both $X \bar{v}$ and $Y \bar{v}$ are in $\mathbf{Z}^{n}$ and hence so is $(A X+B Y) \bar{v}$; then $\bar{v} \in R_{p}(A X+B Y)$.

For the next theorem, we use the notation of Theorem 4.1(g).

4.6. TheOrem. Let $X \in \mathbf{Z}[C]$ be such that $\operatorname{ker}_{p} X=W_{i}^{t_{i}}$ for some $i, 1 \leqslant i \leqslant r$. Put $W=W_{i}, t=t_{i}, k=k_{i}$.

(a) There exist positive integers $s_{1} \leqslant s_{2} \leqslant \cdots \leqslant s_{t}$ such that the p-Smith form of $X$ has the form:

$$
S_{p}(X)=\operatorname{diag}(1,1, \ldots, 1, \underbrace{p^{s_{1}}, \ldots, p^{s_{1}}}_{k}, \ldots, \underbrace{p^{s_{t}} \cdots p^{s_{t}}}_{k}) .
$$

(b) For each $i=1,2, \ldots, t$,

$$
s_{t-i+1}=\max \left\{s:\left(1 / p^{s}\right) \bar{a} \in R_{p}(X), \bar{a} \in W^{i}-W^{i-1}\right\},
$$

and if $V=W^{i}-W^{i-1}$, then $R_{V}(X)$ contains $k$ independent $p^{s_{t-i+1}}$-points.

(c) $R_{p}(X)$ does not contain any elements of order higher than $p^{s_{t}}$.

(d) $R_{W}(X)$ contains $k$ independent $p^{s_{-} \text {-points. }}$

Proof. (a) There exist unimodular integral matrices $P, Q$ (not necessarily in $\mathbf{Z}[C]$ ) so that $P X Q=S(X)$, the Smith form of $X$. Then $X$ and $S(X)$ have the same $p$-nullity $t k=m$, so the $p$-Smith form is

$$
S_{p}(X)=\operatorname{diag}\left(1,1, \ldots, 1, p^{r_{1}}, p^{r_{2}}, \ldots, p^{r_{m}}\right),
$$

where the $r_{i}$ are positive integers, $0<r_{1} \leqslant r_{2} \leqslant \cdots \leqslant r_{m}$.

As is shown in [1], a basis for $R(X)$ consists of the columns of the matrix $Q \cdot S(X)^{-1}$ (reduced modulo 1 , of course); hence, a basis for $R_{p}(X)$ is given by the last $m$ columns of $Q \cdot S_{p}(X)^{-1}$, namely

$$
\left(1 / p^{r_{1}}\right) Q_{n-m+1}, \ldots,\left(1 / p^{r_{m}}\right) Q_{n}
$$

(where $Q_{i}$ is the $i$ th column of $Q$ ). By Lemma 4.3, $\left\{Q_{i} ; i=n-m+1, \ldots, n\right\} \subseteq$ $\operatorname{ker}_{p} X$ and since $Q$ is unimodular, these vectors are independent in $\mathbf{Z}_{p}^{n}$. Thus, this set is a basis for $\operatorname{ker}_{p} X$.

Suppose now that $Q_{n} \in W^{j}-W^{j-1}\left(1 \leqslant j \leqslant t ; W^{0}=\{0\}\right)$. Then $Q_{n}$ is a cyclic vector for $W^{j}$, that is, the set

$$
\left\{C^{i}\left(Q_{n}\right): i=0, \ldots, j k-1\right\}
$$

is a basis for $W^{j}$, and, in particular, for $0 \leqslant i<j k, C^{i}\left(Q_{n}\right) \not \equiv 0(\bmod p)$. Since $X \in \mathbf{Z}[C]$, and $\left(1 / p^{r_{m}}\right) Q_{n} \in R_{p}(X)$, we have

$$
X\left(\left(1 / p^{r_{m}}\right) C^{i}\left(Q_{n}\right)\right)=C^{i} X\left(\left(1 / p^{r_{m}}\right) Q_{n}\right) \in \mathbf{Z}_{n},
$$

so that all these vectors are $p^{r_{m}}$-points for $X$. 
Since the set $\left\{\left(1 / p^{r_{i}}\right) Q_{n-m+i} ; i=1,2, \ldots, m\right\}$ is a basis for $R_{p}(X)$, we have the equality (in $\mathbf{Q}^{n}$ ):

$$
\left(1 / p^{r_{m}}\right) C^{i}\left(Q_{n}\right)=\sum_{u=1}^{m} n_{u}\left(1 / p^{r_{u}}\right) Q_{n-m+u}+\bar{m} \quad\left(n_{u} \in \mathbf{Z} \text { and } \bar{m} \in \mathbf{Z}^{n}\right) .
$$

From this

$$
C^{i}\left(Q_{n}\right)=\sum_{u=1}^{m} n_{u}\left(p^{r_{m}-r_{u}}\right) Q_{n-m+u}+p^{r_{m}} \bar{m}
$$

We have $j k$ vectors $C^{i}\left(Q_{n}\right)$, independent $\bmod p$, written as linear combinations $(\bmod p)$ of those $Q_{n-m+u}$ such that $r_{m}-r_{u}=0$; hence, the number of these must be at least $j k$. Since the $r_{i}$ are in increasing order, we have the last $j k \geqslant k$ of the $r_{i}=r_{m}$.

The same argument now shows that if any of $Q_{n-j k+1}, \ldots, Q_{n}$ were in some $W^{u}-W^{u-1}$, then we would have the last $u k$ of the $r_{i}=r_{m}$. Thus, suppose that all of $Q_{n-j k+1}, \ldots, Q_{n}$ are in $W^{j}$. We have $j k$ independent vectors in a space of dimension $j k$, so they are a basis for $W^{j}$. It follows from the independence of the set $\left\{Q_{i}\right\}$ that none of $Q_{n-m+1}, \ldots, Q_{n-j k}$ are in $W^{j}$ (we are assuming now that $j<t$; of course, if $j=t$, we are done).

Suppose that $Q_{n-j k} \in W^{a}-W^{a-1}, a>j$. Put $r=r_{m-j k}$. The set $\left\{C^{u}\left(Q_{n-j k}\right)\right.$ : $u=0,1, \ldots,(a-j) k-1\}$ is an independent set in $W^{a}-W^{j}$, and as before, we have a set of $p^{r}$-points:

$$
\left(1 / p^{r}\right) C^{u}\left(Q_{n-j k}\right) \in R_{p}(X) ; \quad u=0, \ldots,(a-j) k-1 .
$$

Then we can write

$$
\begin{aligned}
\left(1 / p^{r}\right) C^{u}\left(Q_{n-j k}\right)= & \sum_{s=1}^{m-j k} n_{s}\left(1 / p^{r_{s}}\right) Q_{n-m+s} \\
& +\sum_{s=m-j k+1}^{m} n_{s}\left(1 / p^{r_{m}}\right) Q_{n-m+s}+\bar{m}
\end{aligned}
$$

( $n_{s} \in \mathbf{Z}$ and $\bar{m} \in \mathbf{Z}^{n}$ ), and from this

(*) $\quad C^{u}\left(Q_{n-j k}\right)=\sum_{s=1}^{m-j k} n_{s}\left(p^{r-r_{s}}\right) Q_{n-m+s}+\sum_{s=m-j k+1}^{m} n_{s}\left(1 / p^{r_{m}-r}\right) Q_{n-m+s}+p^{r} \bar{m}$.

The middle term on the right must be integral, since all the other terms are. But the $Q_{i}$ are columns of a unimodular matrix, so this implies that all coefficients of the middle term are integral: $p^{r_{m}-r}$ divides $n_{s}, s=m-j k+1, \ldots, m$.

Next we have (in $\left.\mathbf{Z}_{p}^{n}\right) f_{i}(C)^{j}\left(W^{j}\right)=\{0\}$ while $f_{i}(C)^{j}\left(W^{a}\right)$ is a space of dimension $(a-j) k$, namely $W^{a-j}$. Applying $f_{i}(C)^{j}$ to $(*)$, we have

$$
f_{i}(C)^{j}\left(C^{u}\left(Q_{n-j k}\right)\right) \equiv \sum_{s=1}^{m-j k} n_{s} p^{r-r_{s}} Q_{n-m+s}(\bmod p)
$$

since $Q_{s} \in W^{j}$ for $s>n-j k$. Now, as before, we must have at least $(a-j) k$ of the values $r-r_{s}=0$, since the set

$$
\left\{f_{i}(C)^{j}\left(C^{u}\left(Q_{n-j k}\right)\right): u=0,1, \ldots,(a-j) k-1\right\}
$$

is independent $\bmod p$. By the ordering of the $r_{i}$, the last $(a-j) k$ of the integers $r_{1}, \ldots, r_{m-j k}$ are equal to $r=r_{m-j k}$. Continuing this process, we eventually arrive at (a), as required. 
(b) Let $j_{1}, \ldots, j_{s}$ be the indices $i$ so that $s_{i} \neq s_{i+1}(1 \leqslant s \leqslant t) ; j_{s}=t$. Then the $p$-Smith form is divided into $s$ segments of like powers of $p$, where the $i$ th segment has length $k\left(j_{i}-j_{i-1}\right)$. Group the last $m$ columns of $Q$ correspondingly, into $s$ sets $A_{1}, \ldots, A_{s}$; e.g., $A_{s}$ consists of the last $k\left(j_{s}-j_{s-1}\right)$ columns of $Q$. It is seen in the proof of (a) that $s_{i}<s_{i+1}$ is only possible in case $A_{i+1} \cup \cdots \cup A_{s}$ is a basis for $W^{j}$ where $j=j_{s}-j_{i}$. Thus, $A_{s}$ is a basis for $W^{j_{s}}, A_{s-1}$ is an independent set of cardinality $k\left(j_{s-1}\right)$ in $W^{a}-W^{b}$, where $a=j_{s-1}+j_{s}$ and $b=j_{s}$; and so on.

If $\bar{v} \in W^{a}-W^{a-1}$ for some $a$, then there exists some $i$ so that $\bar{v}$ is in the span of $A_{i} \cup \cdots \cup A_{s}$, but not of $A_{i+1} \cup \cdots \cup A_{s}$. The span of $A_{i} \cup \cdots \cup A_{s}$ contains $W^{b}-W^{b-1}$, where $b=j_{i}+\cdots+j_{s}$. Then

$$
f_{i}(C)^{b-a}\left(W^{b}-W^{b-1}\right)=W^{a}-W^{a-1} .
$$

As in the proof of (a), since $R_{W^{b}}(X)$ has a basis of elements all of whose orders are $\geqslant p^{s_{i}}$, then so does $R_{W^{a}}(X)$. Put $V=W^{a}-W^{a-1}$; then $R_{V}(X)$ must contain $k$

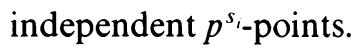

We must now show that $R_{V}(X)$ contains no elements of higher order. Let $p^{r_{i}}$ denote the power of $p$ corresponding to column $Q_{i}$ of $Q$. Suppose that $s>s_{i}$, that $\left(1 / p^{s}\right) \bar{v} \in R_{p}(X)$ and $\bar{v}$ is in the span of $A_{i} \cup \cdots \cup A_{s}$ but not of $A_{i+1} \cup \cdots \cup A_{s}$. We can write

$$
\left(1 / p^{s}\right) \bar{v}=\sum_{r_{i} \leqslant s_{i}} n_{i}\left(1 / p^{r_{i}}\right) Q_{i}+\sum_{r_{i}>s_{i}} m_{i}\left(1 / p^{r_{i}}\right) Q_{i}+\bar{m}
$$

$\left(m_{i}, n_{i} \in \mathbf{Z}\right.$ and $\left.\bar{m} \in \mathbf{Z}^{n}\right)$. Then

$$
\bar{v}=\sum_{r_{i} \leqslant s_{i}} n_{i}\left(p^{s-r_{i}}\right) Q_{i}+\sum_{r_{i}>s_{i}} m_{i}\left(1 / p^{r_{i}-s}\right) Q_{i}+p^{s} \bar{m}
$$

As before, we must have $p^{r_{i}-s}$ dividing each $m_{i}$. But $s>s_{i}$ implies $p^{s-r_{i}} \geqslant p$ for all $r_{i} \leqslant s_{i}$; thus mod $p$, we have $\bar{v}$ in the span of those $Q_{i}$ with corresponding $r_{i} \geqslant s$, that is, in the span of $A_{i+1} \cup \cdots \cup A_{s}$, a contradiction.

Statements (c) and (d) follow from the fact that $W$ is always in the span of $A_{s}$.

This completes the proof.

The next two results indicate how a knowledge of the group $R(X)$ may be helpful in factorization questions.

4.7. TheOREM. Let $A, B \in \mathbf{Z}_{n \times n}$ be nonsingular. Then $R_{p}(A) \subseteq R_{p}(B)$ if and only if there is some integer $k$ such that $(k, p)=1$ and an integral matrix $Y$ so that $k B=Y A$.

Proof. $R(A)$ is generated by the columns of $A^{-1}$ reduced modulo 1 . We can write $A^{-1}=\left(1 / d_{n}\right) D$ where $d_{n}$ is the largest invariant factor of $A$ and $D$ is integral. Suppose $p^{r} \| d_{n}$. Then $R_{p}(A)$ is generated by the columns of $\left(1 / p^{r}\right) D$, reduced modulo 1. Put $d_{n}=k p^{r}$. Clearly, $R_{p}(A) \subseteq R_{p}(B)$ if and only if $B\left(\left(1 / p^{r}\right) D\right)=Y$ is integral, and the result follows.

4.8. TheOREM. Let $f_{i}(x)$ be an irreducible factor of $f(x) \bmod p$, of degree $k_{i}=k$, with $f_{i}(x)^{e_{i}} \| f(x)$. Put $A=f_{i}(C)$ and $W=\operatorname{ker}_{p}(A)$. Suppose that $R_{p}(A)=R_{W}(A)$ does not contain any $p^{2}$-points. Then,

(a) $R_{p}(A)=(1 / p) W$ and $|A|=p^{k} a$ with $(a, p)=1$.

(b) $R_{p}\left(A^{t}\right)=(1 / p) W^{t}$ for $t=1,2, \ldots, e_{i}$. 
(c) If $B=h(C) \not \equiv 0(\bmod p)$ and if $W \subseteq \operatorname{ker}_{p} B$, then there exist integers a with $(a, p)=1$ and $t>0$, and an integral polynomial $k(x)$ such that

$$
W \cap \operatorname{ker}_{p} k(C)=\{0\} \text { and } a B=A^{t} k(C) .
$$

(d) In (c), if $B$ has a $p^{2}$-point for $W$, then $t \geqslant e_{i}+1$.

Proof. (a) By Lemma 4.4, $(1 / p) W \subseteq R_{p}(A)$; by assumption, $A$ has no $p^{2}$-points for $W$, hence $(1 / p) W=R_{W}(A)=R_{p}(A)$. Then, by Theorem 4.6, the $p$-Smith form of $A$ has $\operatorname{dim} W=k$ entries on its diagonal equal to $p$, and the rest equal 1 . Then $|A|=p^{k} a$ for some integer $a$ with $(a, p)=1$.

(b) By Theorem 4.1(f) the dimension of $\operatorname{ker}_{p} A^{t}$ for $1 \leqslant t \leqslant e_{i}$ is $t k$ (the degree of $\left.f_{i}(x)^{t}\right)$; so $t k$ is the $p$-nullity of $A^{t}$. But $\left|A^{t}\right|=p^{k t} a^{t}$ and so the $p$-Smith form of $A^{t}$ must have $t k$ entries equal $p$ and the rest equal 1 . Then, $A^{t}$ has no $p^{2}$-points, and (b) follows as in (a).

(c) This follows from a finite number of applications of Theorem 4.7, since whenever $W \subseteq \operatorname{ker}_{p} B$, then by (a), $R_{p}(A) \subseteq R_{p}(B)$.

(d) Let $B$ be as in (c) above, and suppose $1 \leqslant t \leqslant e_{i}$. Put $B_{1}=k(C)$, and suppose that there is a $p^{2}$-point for $B$ in $W$, say $\left(1 / p^{2}\right) \bar{v}, \vec{v} \not \equiv 0(\bmod p)$. Since $W \cap \operatorname{ker}_{p} B_{1}$ $=\{0\}$, then $B_{1} \bar{v} \neq 0(\bmod p)$ and since $B_{1}=k(C)$ and $W$ is a $C$-invariant subspace, then $B_{1} \bar{v} \in W$. Then, we have

$$
a B\left(1 / p^{2}\right) \bar{v}=A^{t}\left(1 / p^{2}\right) B_{1} \bar{v} \in \mathbf{Z}^{n},
$$

contradicting the fact that for $1 \leqslant t \leqslant e_{i}, A^{t}$ has no $p^{2}$-points in $W$. Thus, $t \geqslant e_{i}+1$ as required.

We conclude this section with

4.9. Theorem. Let $A=g(C) \in \mathbf{Z}[C]$ with invariant factors $d_{1}, d_{2}, \ldots, d_{n}$. Suppose $A \neq 0$, so that $A$ is nonsingular. Then adj $A=d_{1} d_{2} \cdots d_{n-1} B$, where $B=h(C) \in$ $\mathbf{Z}[C]$ and $B \neq 0(\bmod p)$ for every prime $p$ dividing $d_{n}$.

Proof. Let the characteristic polynomial for $A$ be $a_{0}+a_{1} x+\cdots+x^{n}$. Then

$$
A^{-1}=(1 /|A|) \operatorname{adj} A=\left(-1 / a_{0}\right)\left(A^{n-1}+a_{n-1} A^{n-2}+\cdots+a_{1} I\right) .
$$

We have $a_{0}=(-1)^{n}|A|, \pm|A|=d_{1} d_{2} \cdots d_{n}$, and $d_{n}$ is the smallest positive integer such that $d_{n} A^{-1}$ is integral. Then

$$
\operatorname{adj} A= \pm\left(A^{n-1}+\cdots+a_{1} I\right) \equiv 0\left(\bmod d_{1} d_{2} \cdots d_{n-1}\right) .
$$

Now $A=g(C)$, so we can write $\operatorname{adj} A=k(C)$ for some $k(x) \in \mathbf{Z}[x]$. Since $C$ is a companion matrix, then for $i=0,1, \ldots, n-1$, the first column of $C^{i}$ is $\operatorname{col}(0, \ldots, 0,1,0, \ldots$,$) with the 1$ in the $(i+1)$ st place. Then, the first column of $k(C)$ consists of the coefficients of $k(x)$, and so all these coefficients are divisible by $d_{1} \cdots d_{n-1}$. So $k(x)=d_{1} \cdots d_{n-1} h(x)$ for $h(x) \in \mathbf{Z}[x]$, and we have $B=h(C)$. Since $d_{n}$ is the smallest positive integer so that $d_{n} A^{-1}=B$ is integral, then $B \neq \equiv$ $(\bmod p)$ for any prime $p \mid d_{n}$.

Example. Let $n=3$ and $p$ be a fixed prime, $X=g(C), g(x) \in \mathbf{Z}[x]$. If $S_{p}(X)=$ $\operatorname{diag}\left(1,1, p^{t}\right)$ (i.e., if $X$ has $p$-rank 2), then $g(x)$ is divisible by a linear factor of $f(x)$ $(\bmod p)$. If $S_{p}(X)=\operatorname{diag}\left(1, p^{t}, p^{t}\right)$, then $g(x)$ is either divisible by two linear factors of $f(x)$ or by an irreducible quadratic factor of $f(x)(\bmod p)$. If $S_{p}(X)=$ $\operatorname{diag}\left(1, p^{u}, p^{v}\right)$ and $0<u<v$, then $g(x)$ is divisible by two linear factors of $f(x)$ 
$(\bmod p)$, and not by an irreducible quadratic factor. Of course, if $S_{p}(X)=$ $\operatorname{diag}\left(p^{u}, p^{v}, p^{t}\right)$, then $X \equiv 0 \bmod p$ anyway, and $g(x)$ is divisible by $f(x) \bmod p$.

In the next section it is shown that much stronger statements can be made from a knowledge of $S_{p}(X)$ and $R_{p}(X)$.

5. Powers. Throughout this section, $p$ is a fixed prime, and

$$
f(x)=\prod_{i=1}^{r} f_{i}(x)^{e_{i}}(\bmod p) ; \quad \operatorname{deg} f_{i}(x)=k_{i}
$$

is the factorization of $f(x)$ into prime factors in $\mathbf{Z}_{p}[x]$. We let $W_{i}^{t}=\operatorname{ker}_{p} f_{i}(C)^{t}$; $W_{i}^{1}=W_{i}$; all polynomials will be assumed to have coefficients in $\mathbf{Z}$ (or $\mathbf{Z}_{p}$, according to context).

5.2. Definition. If $X=g(C)$ and $W_{i} \subseteq \operatorname{ker}_{p}(X)$, and if

$$
t=\max \left\{j:\left(1 / p^{j}\right) \bar{a} \in R_{W_{i}}(X), \bar{a} \not \equiv 0 \bmod p\right\},
$$

then we say that $X$ has power $t$ for $W_{i}$. Now put

$$
j_{i}=\min \left\{t: t \text { is the power for } W_{i} \text { for some } X=g(C) \text { where } R_{p}(X)=R_{W_{i}}(X)\right\} \text {. }
$$

Then we say that $j_{i}$ is the least power for $W_{i}$, or for $f_{i}(x)$.

Finally, we say that $f_{i}(x)$ is an honest factor (of $f(x)$, mod $p$ ) provided the integer $f_{i}(\mathbf{r})$ (i ecall $\mathbf{r}$ is a root of $f(x)$ in $\mathbf{C}$ ) satisfies

$$
(p)=\left(f_{i}(\mathbf{r}), p\right)^{e_{i}} V
$$

for some ideal $V$ which is relatively prime to $\left(f_{i}(\mathbf{r}), p\right)$; that is, the $p$-ideal for $f_{i}(\mathbf{r})$ actually divides $(p)$ to the exact exponent $e_{i}$.

We shall see that the least power for $W_{i}$ determines the honesty of $f_{i}(x)$; that it is possible for some $f_{i}$ to be honest and not others; that if all $f_{i}$ are honest, then (5.1) yields the complete prime factorization for the ideal $(p)$ in $\mathscr{R}$, and finally, that a dishonest factor may be used to construct an integer $\beta \in \mathscr{R}$ so that $\beta / p \in \mathscr{R}$ also.

The hypotheses required for the results in this section may appear rather technical and difficult of application. We remedy this situation in the next section, where it is seen that the necessary conditions may be decided in a constructive way (which is simpler than one might expect).

We shall need

5.3. Lemma. (a) Let $u_{i}=f_{i}(\mathbf{r}) \in \mathscr{R}$. Then, the ideals $\left(u_{i}, p\right)$ and $\left(u_{j}, p\right)$ where $i \neq j$, are relatively prime.

(b) We may always suppose without loss of generality, that $f_{i}(C)$ has the least power $j$ for $f_{i}(x)$, hence $S_{p}\left(f_{i}(C)\right)=\operatorname{diag}\left(1, \ldots, p^{j}, \ldots, p^{j}\right)$ with determinant $p^{k j}$.

Proof. (a) This follows from the fact that, for $i \neq j$, the polynomials $f_{i}(x)$ and $f_{j}(x)$ are relatively prime in $\mathbf{Z}_{p}[x]$.

(b) Put $W=W_{i}, k=k_{i}$. Suppose $g(C)$ has $\operatorname{ker}_{p} g(C)=W$ and power $j$ for $W$. Then, we must have $g(x)=q(x) f_{i}(x)+\operatorname{pr}(x)$, where $\operatorname{gcd}(q(x), f(x))=1(\bmod p)$; hence, we can write

$$
\begin{aligned}
a(x) q(x)+b(x) f(x) & \equiv 1(\bmod p), \\
a(x) g(x) & \equiv f_{i}(x)(\bmod p, f(x)), \\
a(C) g(C) & =f_{i}(C)+p k(C),
\end{aligned}
$$


for some $k(x) \in \mathbf{Z}[x]$. Since $\operatorname{gcd}(a(x), f(x))=1$, then

$$
R_{p}(a(C) g(C))=R_{p}(g(C))=R_{p}\left(f_{i}(C)+p k(C)\right) .
$$

Then, in the factorization (5.1), we can replace the factor $f_{i}(x)$ by the factor $f_{i}(x)+p k(x)$. By Theorem 4.6 we get the $p$-Smith form.

5.4. THEOREM. If $f_{i}(x)$ has least power 1 , then it is honest.

Proof. Put $A=g(C)$ with $R_{p}(A)=R_{W_{i}}(A)=(1 / p) W_{i}$. Then $\operatorname{gcd}(f(x), g(x))=$ $f_{i}(x) \bmod p$. By Theorem 4.8, $|A|=p^{k_{\prime}} a$ where $(a, p)=1$. By Theorem 4.9, we have adj $A=p^{k_{i}-1} B$, where $B=h(C)$ for some $h(x) \in \mathbf{Z}[x], B \neq \equiv(\bmod p)$ and $A B=$ $p a I_{n}$. Since $A B \equiv 0(\bmod p)$ then $f(x) / f_{i}(x)$ divides $h(x)(\bmod p)$ and since $B \neq \equiv$ $(\bmod p), f(x)+h(x)$. Then, by Theorem 4.8 , we have some integer $b$ with $(b, p)=1$, and $k(x) \in \mathbf{Z}[x]$, so that

$$
b B=A^{e_{i}-1} k(C) \text { and } W_{i} \cap \operatorname{ker}_{p} k(C)=\{0\} .
$$

Put $\alpha=g(\mathbf{r})$ and $\beta=k(\mathbf{r})$; by Lemma 5.3, $(\alpha, p)$ and $(\beta, p)$ are relatively prime. We have $b A B=A^{e_{i}} k(C)=p a b I_{n}$, and so in $\mathscr{R}$,

$$
\alpha^{e_{i}} \beta=p a b .
$$

Since $(a b, p)=1$, then $(\alpha, p)^{e_{i}} \|(p)$ as required.

The next result implies that any nonrepeated factor is honest.

5.5. THEOREM. If the least power $j_{i}$ for $W_{i}$ is $\geqslant 2$, then $e_{i} \geqslant 2$.

Proof. Assume that $A=f_{i}(C)$ has the least power $j$ and put $W=W_{i}$. Write $f(x)=f_{i}(x) h(x)+r(x)$, where either $r(x)=0$ or $0 \leqslant \operatorname{deg} r(x)<\operatorname{deg} f_{i}(x)$.

If $r(x)=0$, then $f_{i}(x)=f(x)$ is irreducible $\bmod p ;|X| \equiv 0(\bmod p)$ if and only if $X \equiv 0 \bmod p$ for any $X=g(C)$, and the least power $j=1$ trivially.

If $r(x) \neq 0$, then since $f_{i}(x) \mid f(x) \bmod p$, we can write $r(x)=p^{t} k(x)$ where $t \geqslant 1$ and $k(x) \neq \equiv(p)$. Since $\operatorname{deg} k(x)<\operatorname{deg} f_{i}(x)$, we have $W \cap \operatorname{ker}_{p} k(C)=\{0\}$ by Lemma 4.4. Since $C$ is the companion matrix of $f(x)$,

$$
f(C)=0=f_{i}(C) h(C)+p^{t} k(C), \quad f_{i}(C) h(C)=-p^{t} k(C) .
$$

There is a $p^{j}$-point in $R_{W}(A)$, say $\left(1 / p^{j}\right) \bar{a}$, and $k(C) \bar{a} \not \equiv 0 \bmod p$. But then

$$
-k(C)\left(1 / p^{j-t}\right) \bar{a}=h(C) f_{i}(C)\left(1 / p^{j}\right) \bar{a} \in \mathbf{Z}^{n}
$$

implies $j \leqslant t$.

Now put $g(x)=f_{i}(x)+p$, and $f(x)=g(x) h_{1}(x)+p^{m} k_{1}(x)$. As above, and using the minimality of $j$, we have $m \geqslant j$. Then,

$$
\begin{aligned}
& f_{i}(x) h(x)+p^{t} k(x)-\left(f_{i}(x)+p\right) h_{1}(x)-p^{m} k_{1}(x)=0, \\
& f_{i}(x)\left[h(x)-h_{1}(x)\right]-p h_{1}(x)+p^{t} k(x)-p^{m} k_{1}(x)=0 .
\end{aligned}
$$

Since $R_{W}(A) \subseteq R_{p}\left(p^{j} I\right)$, it follows from Lemma 4.5 that $R_{W}(A) \subseteq R_{W}\left(p h_{1}(C)\right)$. The least power $j$ for $W$ is greater than 1 , and all terms other than $p h_{1}(C)$ have a common $p^{j}$-point, so $p h_{1}(C)$ must have at least a $p^{2}$-point for $W$ (Lemma 4.5). Then $h_{1}(C)$ must have a $p$-point for $W$, and then $W \subseteq \operatorname{ker}_{p} h_{1}(C)$ (Lemma 4.4). From this, $f_{i}(x) \mid h_{1}(x) \bmod p\left(\right.$ Lemma 4.4); of course $h(x) \equiv h_{1}(x) \bmod p$, and so $f_{i}(x) \mid h(x)$ $\bmod p$. Then $f_{i}(x)$ is a repeated factor of $f(x) \bmod p$, and this completes the proof. 
Since any nonrepeated factor is honest, it is not difficult to find examples for $f(x)$ with some factors honest and some not (see Section 6).

The remainder of this section is devoted to establishing the following:

There exists an integer $\beta=g(\mathbf{r}) \in \mathbf{Z}[\mathbf{r}]$ with $g(x) \not \equiv 0(\bmod p)$ and $\beta / p \in \mathscr{R}$ if and only if at least one of the least powers for the irreducible factors $f_{i}(x)$ of $f(x) \bmod p$, is greater than 1 .

In fact, we shall show that each $f_{i}(x)$ with least power $>1$, gives rise to such a $\beta$. We begin with a necessary condition for $\beta / p \in \mathscr{R}$.

5.6. LemMA. Let $X=g(C)$ and put $y=g(\mathbf{r}) \in \mathscr{R}$. If $y / p \in \mathscr{R}$, then necessarily

$$
f_{1}(x) f_{2}(x) \cdots f_{r}(x) \mid g(x)(\bmod p) \text {. }
$$

Proof. Suppose $y / p$ is an integer. This is to say that the characteristic polynomial of $(1 / p) X$ has coefficients in $\mathbf{Z}$. Then the characteristic polynomial of $X$ itself must have the form:

$$
|\lambda I-X|=\sum_{i=0}^{n-1} \lambda^{n-i} p^{i} a_{n-i} \quad\left(a_{n}=1, a_{i} \in \mathbf{Z}\right) .
$$

Then over $\mathbf{Z}_{p}, X$ is nilpotent. But $X=g(C)$ is nilpotent over $\mathbf{Z}_{p}$ if and only if $f_{1}(x) \cdots f_{r}(x) \mid g(x) \bmod p$.

We now prove half of the statement $(*)$.

5.7. THEOREM. If every $f_{i}(x)(i=1,2, \ldots, r)$ has least power $j_{i}=1$, and if $\beta=g(\mathbf{r})$ $\in \mathbf{Z}[\mathbf{r}]$, where $\beta / p \in \mathscr{R}$, then $g(x) \equiv 0 \bmod p$.

Proof. Assume to the contrary, that $X=g(C), g(x) \not \equiv 0 \bmod p$, and $\beta=g(\mathbf{r})$ satisfies $\beta / p \in \mathscr{R}$. We may assume that $A_{i}=f_{i}(C)$ has power 1 for $W_{i}$. Put $\alpha_{i}=f_{i}(\mathbf{r})$ and $P_{i}=\left(\alpha_{i}, p\right)$. Then,

$$
\begin{gathered}
A_{1}^{e_{1}} A_{2}^{e_{2}} \cdots A_{r}^{e_{r}}=p y I_{n} \quad((y, p)=1, y \in \mathbf{Z}), \\
P_{1}^{e_{1}} \cdots P_{r}^{e_{r}}=(p) .
\end{gathered}
$$

By Lemma 5.6 we have (ignoring factors relatively prime to $p$ )

$$
X=A_{1}^{s_{1}} \cdots A_{r}^{s_{r}} \quad\left(s_{i}>0, i=1, \ldots, r\right) .
$$

If all $s_{i} \geqslant e_{i}$, then $g(x) \equiv 0 \bmod p$, and if all $s_{i}<e_{i}$, then $|X|<p^{n}$; thus our assumptions imply some $s_{i} \geqslant e_{i}$ and some $s_{i}<e_{i}$. For simplicity, suppose $s_{r}<e_{r}$ and all other $s_{i} \geqslant e_{i}$. We have

$$
\begin{aligned}
& (1 / p) X=(1 / p) A_{1}^{e_{1}} A_{1}^{s_{1}-e_{1}} \cdots A_{r}^{s_{r}} \\
& (y / p) X=\left(A_{2}^{e_{2}} \cdots A_{r}^{e_{r}}\right)^{-1} A_{1}^{s_{1}-e_{1}} A_{2}^{s_{2}} \cdots A_{r}^{s_{r}}=A_{1}^{s_{1}-e_{1}} \cdots A_{r}^{s_{r}-e_{r} .}
\end{aligned}
$$

Now put $u=y \beta / p \in \mathscr{R}$; then we have

$$
u \alpha_{r}^{e_{r}-s_{r}}=\alpha_{1}^{e_{1}-s_{1}} \cdots \alpha_{r-1}^{s_{r-1}}-e_{r-1}
$$

contradicting the fact that the ideals $\left(\alpha_{i}, p\right)$ are pairwise relatively prime (Lemma 5.3).

5.8. COROllaRy. The conditions of Theorem 5.7 imply

(a) $p+(\operatorname{disc} \mathbf{r}) /(\operatorname{disc} \mathscr{R})$.

(b) The factorization (5.1) yields the prime factorization of the ideal ( $p)$; that is, with $P_{i}=\left(f_{i}(\mathbf{r}), p\right)$, then $P_{i}$ is a prime ideal $(i=1, \ldots, r)$ and

$$
p=\prod_{i=1}^{r} P_{i}^{e_{i}}
$$


We now complete the proof of $(*)$ with

5.9. Theorem. Suppose $f_{i}(x)$ has least power $j \geqslant 2$ and that $A=f_{i}(C)$ has power $j$ for $W_{i}$. Define the integral matrix $B$ by:

$$
\operatorname{adj} A=p^{j\left(k_{1}-1\right)} B \text {. }
$$

Then $B=h(C) \in \mathbf{Z}[C]$, where $h(x) \neq \equiv(\bmod p)$ and $h(\mathbf{r}) / p \in \mathscr{R}$.

Proof. We have $A B=p^{j} \cdot y \cdot I_{n}$ with $(y, p)=1$; without loss of generality assume $y=1$. By Theorem 4.9, $B=h(C) \in \mathbf{Z}[C]$ and $h(x) \not \equiv 0(\bmod p)$. Since $j \geqslant 2$, then $e=e_{i} \geqslant 2$ necessarily; since $A B \equiv 0(\bmod p)$, then $f(x) / f_{i}(x) \mid h(x)$ and since $B \neq 0(\bmod p), f_{i}(x)^{e}+h(x) ; f_{i}(x)^{e-1} \| h(x)(\bmod p)$, and we have

$$
h(x)=f_{i}(x)^{e-1} u(x)+p v(x), \quad\left(f_{i}(x), u(x)\right)=1 .
$$

Then we can write $B=A^{e-1} X+p Y$, where $X, Y \in \mathbf{Z}[C]$. Let the integers in $\mathscr{R}$ corresponding to $A, B, X, Y$ be denoted by $\alpha, \beta, x, y$. Then by Lemma $5.3,(\alpha, p)$ and $(x, p)$ are relatively prime. Suppose that in $\mathscr{R}$ the prime factorization of $(p)$ is

$$
(p)=P_{1}^{t_{1}} \cdots P_{v}^{t_{l}} Q_{1}^{u_{1}} \cdots Q_{s}^{u_{s}},
$$

where the $P_{i}$ are the primes dividing $(\alpha, p)$ and the $Q_{i}$ are the primes dividing $(x, p)$. From $\beta=\alpha^{e-1} x+p y$ and $\alpha \beta=p^{j}$ it is clear that $Q_{k}^{u_{k}} \operatorname{divides}(x, p), k=1, \ldots, s$.

Now suppose $(\alpha) \subseteq P_{1}^{a_{1}} \cdots P_{v}^{a^{c}}\left(a_{i}\right.$ maximal) and consider $\alpha^{e} x+p y \alpha=p^{j}$. We have

$$
\left(\alpha^{e} x\right) \subseteq P_{1}^{e a_{1}} \cdots P_{v}^{e a_{r}} ; \quad(\text { py } \alpha) \subseteq P_{1}^{t_{1}+a_{1}} \cdots P_{v}^{t_{t^{r}}+a_{t^{r}}} .
$$

If $e \cdot a_{i}<t_{i}+a_{i}$, then $e a_{i}=j t_{i}$, and $(j-1) t_{i}<a_{i}$. Since $j>1$, then $t_{i}<a_{i}$, and we get $P_{i}^{t_{i}} \mid(\alpha)$. If $e a_{i}>t_{i}+a_{i}$, then $a_{i}=(j-1) t_{i} \geqslant t_{i}$, and again $P_{i}^{t_{i}} \mid(\alpha)$. If $e a_{i}=t_{i}+a_{i}$, then $(e-1) a_{i}=t_{i}$ and $P_{i}^{t^{\prime}} \mid\left(\alpha^{e-1}\right)$. In all cases we have

$$
P_{i}^{t_{i}} \mid\left(\alpha^{e-1}\right) \text { and } Q_{i}^{u_{i}} \mid(x)
$$

and hence, $\alpha^{e-1} x \equiv 0 \bmod p$. Then $\beta=\alpha^{e-1} x+p y=p z$ for some $z$ in $\mathscr{R} ; \beta / p \in \mathscr{R}$ as required.

5.10. Corollary. The conditions of Theorem 5.9 imply that $p \mid(\operatorname{disc} \mathbf{r}) /(\operatorname{disc} \mathscr{R})$.

6. Computational Methods and Examples. Given the factorization

$$
f(x)=\prod_{i=1}^{r} f_{i}(x)^{e_{i}}(\bmod p)
$$

we wish to know whether a factor $f_{i}(x)$ is honest or not. It is if $e_{i}=1$, but if $e_{i}>1$, the general results of Section 5 do not look very helpful. Using these results, however, we can prove some things which, while not perhaps of great theoretical interest, are yet very convenient for computational purposes. We begin with

6.1. THEOREM. Let $j$ be the least power for the irreducible factor $f_{i}(x)$ of $f(x) \bmod p$. Suppose also that $e_{i}>1$. Then $j \geqslant 2$ if and only if $R_{p}\left(f_{i}(C)\right)$ contains a $p^{2}$-point.

Proof. We have $e_{i}>1$, and from Theorem 4.8, if $\operatorname{ker}_{p} A=W$ precisely, for some $A$, and if the least power for $W$ were equal to 1 , then $A$ could not have any $p^{2}$-points for $W$. Hence, if $A$ does have such points, the least power for $W$ must be at least 2 . 
On the other hand, if the least power for $W$ is $\geqslant 2$, then every matrix $A \in \mathbf{Z}[C]$ with $\operatorname{ker}_{p} A=W$ must have $p^{2}$-points for $W$; in particular this applies to $A=f_{i}(C)$.

Procedure. (The procedure described below is not too difficult if $n$ and $p$ are not "too large".) (a) Compute $f_{i}(C)$, working $\bmod p^{2}$; (b) Still working $\bmod p^{2}$, row-reduce $f_{i}(C)$, usually to at least a row-echelon form; (c) If the corresponding system of linear equations has a nontrivial solution vector $\bmod p^{2}$, then this gives a $p^{2}$-point for $f_{i}(C)$, and if there is no solution mod $p^{2}$, then $f_{i}(C)$ has no $p^{2}$-points.

One could also deduce this information from the $p$-Smith form of $f_{i}(C)$, or from its determinant, but these things require more work than row-reduction as above. We give some examples below. The next theorems are occasionally helpful.

6.2. THEOREM. Let $f_{i}(x)$ be an irreducible factor of $f(x)$, and $j=j_{i}$ the least power for $f_{i}(x)$. Then $j \geqslant 2$ if and only if whenever

$$
\begin{aligned}
& f(x)=g(x) h(x)+r(x) \quad(\text { in } \mathbf{Z}[x]), \\
& g(x) \equiv f_{i}(x)(\bmod p), \\
& r(x)=0 \quad \text { or } \quad 0 \leqslant \operatorname{deg} r(x)<\operatorname{deg} g(x),
\end{aligned}
$$

we have $r(x) \equiv 0\left(\bmod p^{2}\right)$.

Proof. See the first part of the proof of Theorem 5.5.

Example. Take $p=3, f(x)=x^{3}-19$. We can write $f(x)=(x-1)\left(x^{2}+x+1\right)$ $-18 ; 18 \equiv 0(\bmod 9)$ is suspicious and one must investigate further. If $p=3$, $f(x)=x^{3}-4$, we write $f(x)=(x-1)\left(x^{2}+x+1\right)-3$; since $3 \neq \equiv(\bmod 9)$ this factorization is honest.

This theorem has a simple corollary which is also useful.

6.3. Corollary. Suppose that for some prime $p$, we have $p^{2}\left|a_{0}, p\right| a_{1}$ (where $\left.f(x)=x^{n}-a_{n-1} x^{n-1}-\cdots-a_{1} x-a_{0}\right) ;$ put $f_{1}(x)=x$. Then $(\bmod p)$ the least power for $f_{1}(x)$ is $\geqslant 2$, and $p \mid(\operatorname{disc} \mathbf{r} / \operatorname{disc} \mathscr{R})$.

6.4. THEOREM. With notation as above, $j \geqslant 2$ if and only if there exist matrices $X$ and $Y$ in $\mathbf{Z}[C]$ such that $\operatorname{ker}_{p} X=\operatorname{ker}_{p} Y=W_{i}=W$ but

$$
R_{W}(X) \nsubseteq R_{W}(Y) \text { and } R_{W}(Y) \nsubseteq R_{W}(X) \text {. }
$$

In fact, $j \geqslant 2$ if and only if $R_{W}(X)$ contains even one point not in $R_{W}(Y)$ or vice versa.

Proof. Suppose the contrary. Let $A=f_{i}(C)$ have the least power $j$ for $W$, and put $B=A+p I_{n}$. Then $\operatorname{ker}_{p} B=\operatorname{ker}_{p} A=W$ and $B$ has power $m \geqslant j$ for $W$. Suppose $R_{p}(A) \subseteq R_{p}(B)$. By Theorem 4.7, there exist an integer $k$ so that $(k, p)=1$ and an integral matrix $Y$ so that $k B A^{-1}=Y=k\left(I_{n}+p A^{-1}\right)$. But since $A$ has power $j$ for $W$, we may write $A^{-1}=\left(1 / p^{j} a\right) D$ where $(a, p)=1$ and $D$ is an integral matrix, $D \not \equiv 0(\bmod P)$. Thus $k\left(I_{n}+p A^{-1}\right)$ cannot be integral since $j \geqslant 2$; a contradiction. Next suppose $R_{p}(B) \subseteq R_{p}(A)$. The order of $R_{p}(B)$ is $p^{k m}$ ( $k$ is the degree of $f_{i}(x)$ ) and the order of $R_{p}(A)$ is $p^{k j}$; since $m \geqslant j$, we must have in this case $m=j$. Then the argument above applies, and this completes the proof.

The following example, which is fairly simple and could doubtlessly be done in many ways, serves to illustrate the methods of this paper. 
6.5. Example. Let $f(x)=x^{4}+x^{3}+9$, with root $\mathbf{r}$. Then $f(x)$ is irreducible since $x^{4}+x^{3}+1$ is irreducible over $\mathbf{Z}_{2}$. We have

$$
\begin{gathered}
C=\left[\begin{array}{cccc}
0 & 0 & 0 & -9 \\
1 & 0 & 0 & 0 \\
0 & 1 & 0 & 0 \\
0 & 0 & 1 & -1
\end{array}\right] ; \\
\left|f^{\prime}(C)\right|=\left|\begin{array}{cccc}
0 & -36 & 9 & -9 \\
0 & 0 & -36 & 0 \\
3 & 0 & 0 & -36 \\
4 & -1 & 1 & -1
\end{array}\right|=9^{3} \times 11 \times 23 .
\end{gathered}
$$

We have $\operatorname{disc} \mathbf{r}=k^{2}$ disc $\mathscr{R}$; evidently $k$ must be either a power of 3 , or 1 . Since $|C|=3^{2}$, we know $3 \mid k$ by Theorem 6.1 ; and by Theorem $5.9(1 / 3)$ adj $C$ represents an algebraic integer. Here, adj $C=-C^{3}-C^{2}$, so $\left(\mathbf{r}^{3}+\mathbf{r}^{2}\right) / 3 \in \mathscr{R}$. It is well-known that $\mathscr{R}$ has an iniegral basis of the form $\left\{1, g_{1}(\mathbf{r}) / d_{1}, g_{2}(\mathbf{r}) / d_{2}, g_{3}(\mathbf{r}) / d_{3}\right\}$, where the $g_{i}(x)$ are monic polynomials of degree $i$, in $\mathbf{Z}[x]$, and $d_{i} \mid d_{i+1}$. We shall find such a basis for $\mathscr{R}$. We have $f(x)=x^{3}(x+1)(\bmod 3)$; by Lemma 5.6 , a necessary condition for $(1 / 3) g(\mathbf{r}) \in \mathscr{R}$ is that $x(x+1)$ divide $g(x)(\bmod 3)$. Hence, if $g(x)=$ $x+a$, then $(1 / 3) g(\mathbf{r}) \notin \mathscr{R}$, and so $g_{1}(x)=x, d_{1}=1$. Next, if $g(x)=x^{2}+s x+t$ $=\left(x^{2}+x\right)+(s-1) x+t$, and if $s-1, t \neq \equiv(\bmod 3)$, then $(1 / 3) g(\mathbf{r}) \notin \mathscr{R}$; thus for $g_{2}(\mathbf{r})$ we need only consider $\left(\mathbf{r}^{2}+\mathbf{r}\right)$. The characteristic polynomial of the matrix

$$
C^{2}+C=\left[\begin{array}{cccc}
0 & 0 & -9 & 0 \\
1 & 0 & 0 & -9 \\
1 & 1 & 0 & 0 \\
0 & 1 & 0 & 0
\end{array}\right]
$$

is $x^{4}+c_{3} x^{3}+c_{2} x^{2}+c_{1} x+c_{0}$, where $c_{4-i}$ is the sum of the principal $i \times i$ minors (except for sign). We find $c_{1}=-9$, not divisible by 27 , so $(1 / 3)\left(\mathbf{r}^{2}+\mathbf{r}\right) \notin \mathscr{R}$; then $g_{2}(\mathbf{r})=\mathbf{r}^{2}$ and $d_{2}=1$. We know that $d_{3}$ is at least 3 ; we inquire whether it may be 9 or more. Consider

$$
g(x)=x^{3}+u x^{2}+s x+t=\left(x^{2}+x\right)(x+u-1)+(s-u+1) x+t .
$$

We require $s-u+1 \equiv 0(\bmod 3)$ and $t \equiv 0(\bmod 3)$. Since $\left(\mathbf{r}^{3}+\mathbf{r}^{2}\right) / 3 \in \mathscr{R}$, then $g(\mathbf{r}) / 3 \in \mathscr{R}$ implies $(u-1)\left(\mathbf{r}^{2}+\mathbf{r}\right) / 3 \in \mathscr{R}$ and from the preceding case we must have $u-1 \equiv 0(\bmod 3)$. Supposing $g(\mathbf{r}) / 3 \in \mathscr{R}$, we may write

$$
\begin{aligned}
g(\mathbf{r}) & =\left(\mathbf{r}^{2}+\mathbf{r}\right)(\mathbf{r}+3 a)+3(b \mathbf{r}+c) \quad(a, b, c \in \mathbf{Z}), \\
\mathbf{r} \times g(\mathbf{r}) & =\left(\mathbf{r}^{4}+\mathbf{r}^{3}\right)+3 a\left(\mathbf{r}^{3}+\mathbf{r}^{2}\right)+3\left(b \mathbf{r}^{2}+c \mathbf{r}\right), \\
9+\mathbf{r} \times g(\mathbf{r}) & =3 a\left(\mathbf{r}^{3}+\mathbf{r}^{2}\right)+3\left(b \mathbf{r}^{2}+c \mathbf{r}\right) .
\end{aligned}
$$

If $(1 / 9) g(\mathbf{r}) \in \mathscr{R}$, then so is

$$
a\left(\mathbf{r}^{3}+\mathbf{r}^{2}\right) / 3+\left(b \mathbf{r}^{2}+c \mathbf{r}\right) / 3 \in \mathscr{R} .
$$

Since $\left(\mathbf{r}^{3}+\mathbf{r}^{2}\right) / 3 \in \mathscr{R}$, then from $(*)\left(b \mathbf{r}^{2}+c \mathbf{r}\right) / 3 \in \mathscr{R}$. But then $b \equiv c \equiv 0$ (mod 3). Reducing coefficients $\bmod 9$, we get

$$
g(\mathbf{r})=\left(\mathbf{r}^{2}+\mathbf{r}\right)(\mathbf{r}+3 a)
$$

where $a$ is one of $0,1,2$. Then for the norm of $g(\mathbf{r})$, we find $\left|C^{2}+C\right|=81$ and $|C+3 a I|=9\left(9 a^{4}-3 a^{3}+1\right)$, so $9^{4}+|g(C)|$. Then $(1 / 9) g(\mathbf{r}) \notin \mathscr{R}$, and we have $d_{3}=3, g_{3}(\mathbf{r})=\left(\mathbf{r}^{3}+\mathbf{r}^{2}\right)$. Finally, we have disc $\mathscr{R}=9^{2} \times 11 \times 23$. 
6.6. Example. Take $n=7, p=5$,

$$
\begin{aligned}
f(x) & =x^{7}+48 x^{6}+27 x^{5}+48 x^{4}-3 x^{3}-3 x+48 \\
& =\left(x^{3}+x+1\right)^{2}(x-2)+25\left(2 x^{6}+x^{5}+2 x^{4}+2\right) .
\end{aligned}
$$

Then $f(x)$ is irreducible by Eisenstein's criterion, and the factorization $\bmod 5$ is suspect. We know that in any case, the factor $x-2(\bmod 5)$ is honest, for it is not repeated. Put $g(x)=x^{3}+x+1$.

In order to use Theorem 6.1, it suffices to work with matrices reduced mod 25 in order to find 25-points for the originals. Below on the left is $A=C^{3}+C+I_{7}(C$ is the companion matrix of $f(x)$ ), and on the right is the reduced row echelon form of $A$; both reduced $\bmod 25$.

$$
\left[\begin{array}{ccccccc}
1 & 0 & 0 & 0 & 2 & 4 & 6 \\
1 & 1 & 0 & 0 & 3 & 8 & 13 \\
0 & 1 & 1 & 0 & 0 & 3 & 8 \\
1 & 0 & 1 & 1 & 3 & 6 & 12 \\
0 & 1 & 0 & 1 & 3 & 7 & 12 \\
0 & 0 & 1 & 0 & -1 & -1 & 1 \\
0 & 0 & 0 & 1 & 2 & 3 & 5
\end{array}\right]
$$

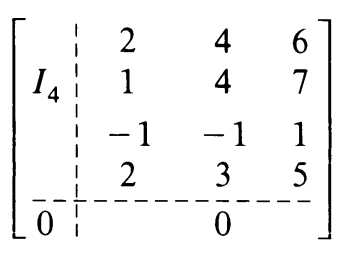

Any vector in the solution set of the right-hand matrix yields a 25-point for $A$, for instance, we find $\bar{v}=(1 / 25) \operatorname{col}(-2,-1,1,2,1,0,0)$ and $g(x)$ is dishonest. For purposes of comparison, consider $\mathrm{A}+5 \mathrm{I}_{7}$; this row-reduces to

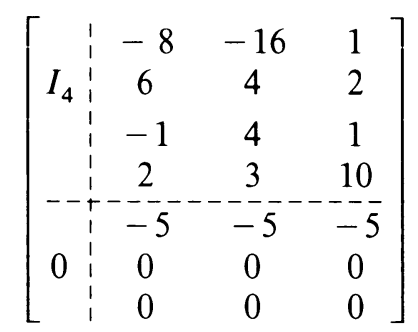

and this solution set gives a 25-point for $A+5 I_{7}$, namely

$$
\bar{w}=(1 / 25) \operatorname{col}(8,2,-5,-1,-1,1,0) .
$$

It suffices to use the $(\bmod 25)$ versions to find that $A \bar{w} \in(1 / 5) \mathbf{Z}^{7}$ and not in $\mathbf{Z}^{7}$.

From the original factorization of $f(x)$ we know $A=g(C)$ has power 2, which must then be the least power; $\operatorname{dim}_{\operatorname{ker}_{5}} A=3$, and $S_{5}(A)=\operatorname{diag}\left(1,1,1,1,5^{2}, 5^{2}, 5^{2}\right)$. Then $\operatorname{adj} A=5^{4} B$ where $B$ is integral and $B \neq \equiv(\bmod 5) ;(1 / 5) B$ represents an algebraic integer, and 5 divides $\operatorname{disc}(\mathbf{r}) / \operatorname{disc}(\mathscr{R})$.

\section{Open Problems.}

7.1. By Lemma 5.6, a necessary condition for $(1 / p) h(C)$ to represent an algebraic integer is that $h(C)$ should be nilpotent $(\bmod p)$. Given $n \geqslant 3$, and supposing the factorization of $f(x)$ is dishonest, is there a $j=j(n)$ so that for any $h(C)$ with index of nilpotency $j(\bmod p),(1 / p) h(C)$ represents an algebraic integer? Does $j=2$ always work?

7.2. If in the factorization 5.1 some $f_{i}(x)$ is honest (but not all), is $\left(f_{i}(\mathbf{r}), p\right)$ a prime ideal? If not, find a counterexample. 
7.3. Suppose some of the $f_{i}(x)$ are honest and some not. The honest factors correspond to relatively prime ideal factors of $(p)$ and each dishonest factor gives rise to a $B$ with $(1 / p) B$ representing an algebraic integer. Is this a new ideal factor of $(p)$ (at least, relatively prime to the honest ones)?

7.4. What is the relation (if any) between the least powers for the $f_{i}(x)$ and the power of $p$ dividing $\operatorname{disc}(\mathbf{r}) / \operatorname{disc}(\mathscr{R})$ ?

7.5. If two dishonest factors have different least powers, do they give rise to different ideal factors of $(p)$ ? (Via Theorem 5.9, that is.)

Department of Mathematics

University of North Carolina at Greensboro

Greensboro, North Carolina 27412

1. Ken BYRD \& Theresa P. VAUGHAN, “A group of integral points in a matrix parallelepiped," Linear Algebra Appl., v. 30, 1980, pp. 155-166.

2. Harvey Cohn, A Classical Invitation to Algebraic Numbers and Class Fields, Springer-Verlag, Berlin and New York, 1978.

3. Kenneth Hoffman \& Ray Kunze, Linear Algebra, Prentice-Hall, Englewood Cliffs, N. J., 1961.

4. Morris Newman, Integral Matrices, Academic Press, New York, 1972. 